\title{
Adaptive Control of Necklace States in a Photonic Crystal Waveguide
}

Emre Yüce, ${ }^{*}, \dagger, \ddagger \odot$ Jin Lian, ${ }^{\dagger, \S}$ Sergei Sokolov, ${ }^{\dagger, \S}$ Jacopo Bertolotti, $^{\perp}$ Sylvain Combrié,, Gaëlle Lehoucq, Alfredo De Rossi," and Allard P. Mosk ${ }^{\dagger, \S}$

${ }^{\dagger}$ Complex Photonic Systems (COPS), MESA+ Institute for Nanotechnology, University of Twente, P.O. Box 217, 7500 AE Enschede, The Netherlands

${ }^{\ddagger}$ Programmable Photonics Group, The Center for Solar Energy Research and Applications (GÜNAM), Department of Physics, Middle East Technical University, 06800 Ankara, Turkey

${ }^{\S}$ Debye Institute for Nanomaterials Science, Utrecht University, PO Box 80000, 3508 TA Utrecht, The Netherlands

${ }^{\perp}$ Physics and Astronomy Department, University of Exeter, Stocker Road, Exeter EX4 4QL, United Kingdom

"Thales Research and Technology, Route Départementale 128, 91767 Palaiseau, France

Supporting Information

ABSTRACT: Resonant cavities with high quality factor and small mode volume provide crucial enhancement of light-matter interactions in nanophotonic devices that transport and process classical and quantum information. The production of functional circuits containing many such cavities remains a major challenge, as inevitable imperfections in the fabrication detune the cavities, which strongly affects functionality such as transmission. In photonic crystal waveguides, intrinsic disorder gives rise to high- $Q$ localized resonances through Anderson localization; however their location and resonance frequencies are completely random, which hampers functionality. We present an adaptive holographic method to gain
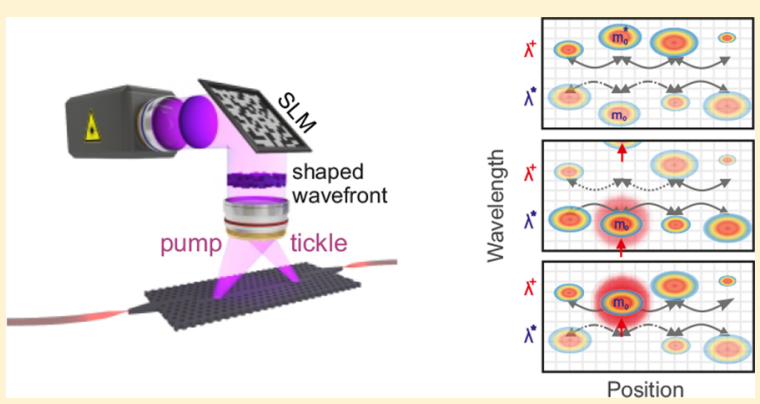
reversible control on these randomly localized modes by locally modifying the refractive index. We show that our method can dynamically form or break highly transmitting necklace states, which is an essential step toward photonic-crystal-based quantum networks and signal processing circuits, as well as slow light applications and fundamental physics.

KEYWORDS: photonic crystals, scattering, Anderson localization, spectroscopy, adaptive control

$\mathrm{D}$ isorder-induced scattering of light is commonly regarded as a loss mechanism as it degrades transmission. ${ }^{1-3}$ However, scattering can also give rise to localized resonant modes, with a potentially very high quality factor. In particular, for photonic crystals close to the band edge of a photonic crystal membrane, the formation of Anderson localized modes ${ }^{4}$ is a natural consequence of intrinsic disorder. ${ }^{1,2,5-10}$ Moreover, when the localization length is shorter than the length of the waveguide, Anderson-localized modes are known to appear. ${ }^{6,711}$ The confinement strength, lifetime, and the spatial profile of these modes are statistically controlled by the dimensionality of the system and the strength of the random scattering. ${ }^{11,12}$ The quality factors, mode volumes, and Purcell enhancement distributions of localized modes in the Anderson localization regime have been successfully modeled in three dimensions in ref 9. In an irreversible manner, light-induced oxidation of the surface was used to control such a single localized mode. ${ }^{13}$ To date, no reversible control of localized resonant modes has been demonstrated, which is an essential step toward programmable photonic circuits that require control over disorder-induced imperfections.
Here, we develop a novel approach, where the high- $Q$ modes generated by the structural randomness are reversibly controlled and coupled into a high-transmission necklace state $^{14}$ We first all-optically locate the relevant Anderson localized modes and subsequently tune their resonance wavelengths independently. By identifying and tuning a link mode, we can form or break necklace states and thereby program the transmitted signal.

A versatile apparatus is used to control the localized modes in our photonic crystal waveguide. ${ }^{15}$ The setup is shown in Figure 1 and consists of a tunable laser at the conventional (C) telecom band and a $405 \mathrm{~nm}$ diode laser that are the sources of the signal and pump light, respectively. The resonance wavelengths of the localized modes are determined from the transmission spectrum. A spatially structured pump beam is generated by a spatial light modulator (SLM) that projects digital holograms. This holographic control of the pump beam enables us to project multiple independently controlled pump spots on the sample, which we use to locate, tune, and perturb

Received: July 27, 2018

Published: September 14, 2018 
a

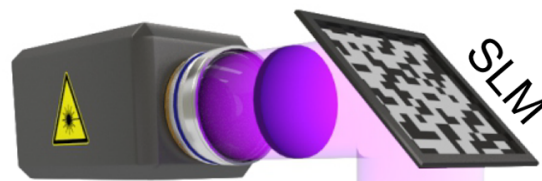

pump: $405 \mathrm{~nm}$
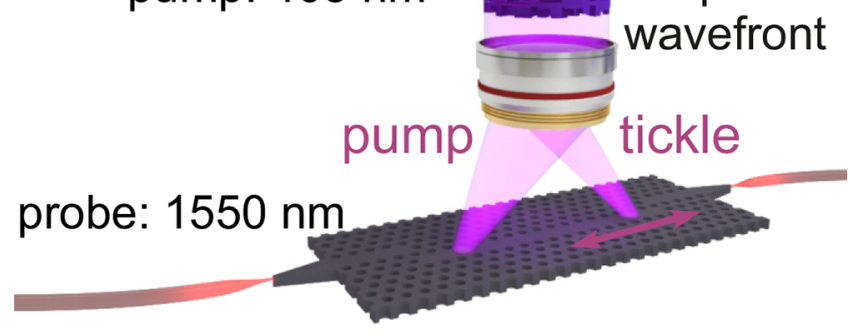

b

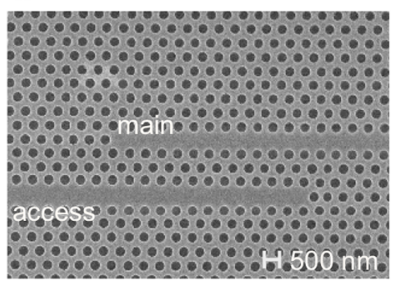

C

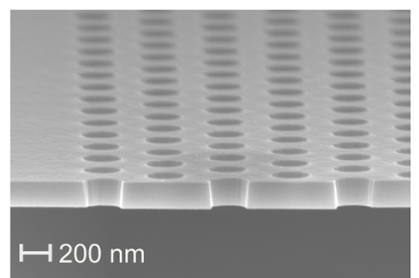

Figure 1. (a) Schematic of the experiment. A narrow-band signal beam is coupled to the GaInP photonic crystal waveguide via a tapered fiber. A second tapered fiber is positioned at the other end of the sample to guide the transmitted signal. The pump beam (violet) is sent at normal incidence to the photonic crystal plane and is spatially structured by a spatial light modulator (SLM) such that multiple spots with different powers are formed on the sample. (b) SEM image of our samples showing the access and main waveguides. (c) Side view showing the membrane structure.

the localized modes. Here, we study the mode profiles using a novel "pump-tickle-probe" strategy, where a first strong

pump beam is used to perturb the modes, and a weak secondary pump beam we designate as "tickle" is used to elucidate the spatial profile of the perturbed modes while inducing negligible further perturbation. The weak $405 \mathrm{~nm}$ "tickle" beam $(16 \mu \mathrm{W}$ and fwhm $\approx 0.96 \mu \mathrm{m})$ introduces a local thermo-optic perturbation $\left(\delta n \approx 10^{-4}\right)$ that effectively shifts the resonance of any mode it spatially overlaps with by up to $\sim 0.4 \mathrm{~nm}$ with a response time of $6 \mu \mathrm{s}$ in nitrogen atmosphere. ${ }^{15}$ Measuring the resonance wavelength shift as a function of the tickle beam position, we infer the position of the localized modes. ${ }^{16}$ Subsequently, we use the strong primary pump beam to tune a targeted mode. In our measurements, the typical pump power is on the order of $100 \mu \mathrm{W}$, which induces a local index change as large as $\delta n \approx 10^{-3}$ through thermooptic perturbation. The width of the thermally induced refractive index profile is about $5 \mu \mathrm{m}$ fwhm. The shift of the resonant frequencies is mainly controlled by the overlap of the pump beam and the spaital profile of the localized mode as well as the pump power. ${ }^{15}$ The pump beam is always focused on the linking modes during the measurements while we spatially scan the tickle beam and collect transmitted light of the probe beam. The probe laser light was coupled to the $\mathrm{PhC}$ waveguide using a polarization-maintaining lensed fiber with an NA of 0.55 . We perform our measurements in a flushed $\mathrm{N}_{2}$ environment to reduce oxidation, which would otherwise result in irreversible changes. ${ }^{15}$

Our photonic crystal sample is a GaInP membrane structure with a membrane thickness of $180 \mathrm{~nm}$, and the lattice constant of our photonic crystal waveguide is $a=485 \mathrm{~nm}$. The width of the main (barrier) waveguide is $W_{0}=0.98 \sqrt{3} a$ and its length is $L=106 a$. The main waveguide is side coupled to two access waveguides with widths $W_{1}=1.1 \sqrt{3} a$ that are positioned at the input and the output facet and serve to couple light in and out of the structure,; see Figure 1(b) and (c). The main
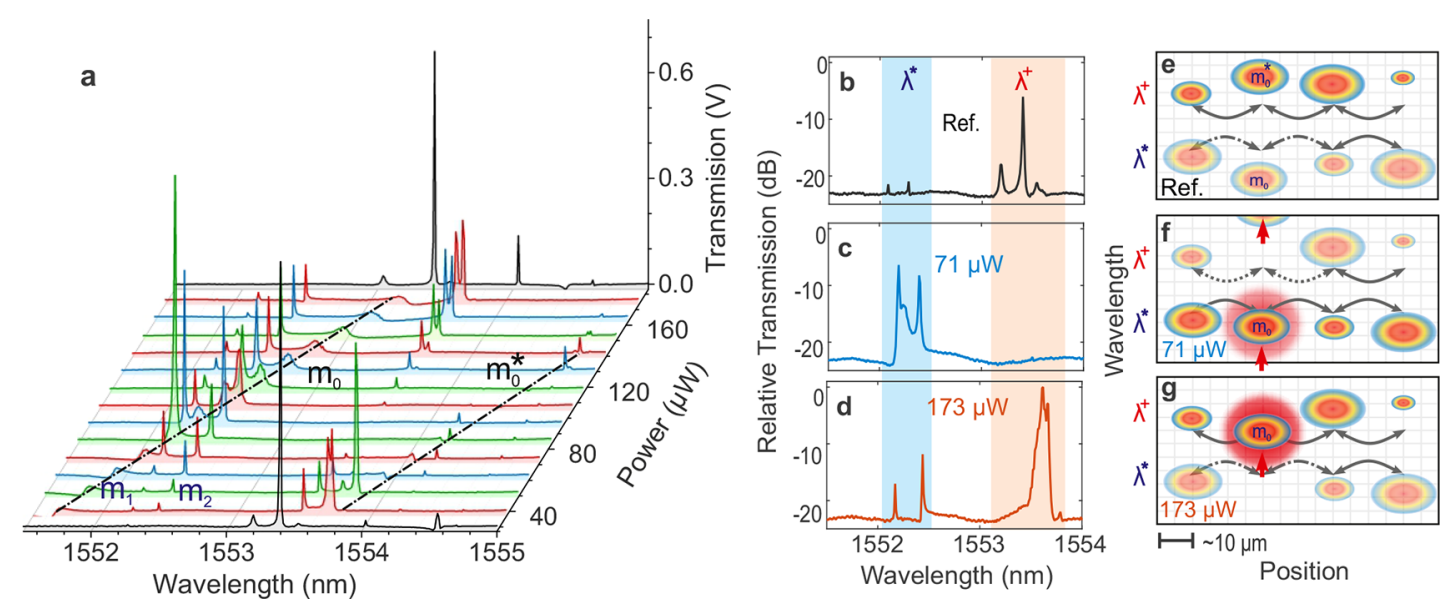

Figure 2. Measured and schematic representation of the localized modes that form a necklace state in a photonic crystal waveguide and the control of the necklace links. (a) Transmission through the GaInP photonic crystal waveguide illustrating the full range of localized modes while the power on the linking modes is scanned between 37 and $173 \mu \mathrm{W}$. The dashed dotted black lines trace the resonance wavelength of the link modes $m_{0}$ and $m_{0}^{*}$. The first and the last black curves are the reference measurements before and after controlling the necklace states, respectively. (b, e) Unperturbed localized modes in a photonic crystal waveguide. At $\lambda^{*}$ light is weakly transmitted due to small spectral overlap of the modes, whereas at $\lambda^{+}$light is transmitted given a larger spectral overlap of the mode $m_{0}^{*}$ with the rest of the necklace. (c, f) The mode $m_{0}$ is locally tuned and thereby shifted in wavelength, which increases its coupling to the rest of the necklace modes at $\lambda^{*}$. Thereby the transmission is increased. The necklace link at $\lambda^{+}$is incomplete given that the mode $m_{0}^{*}$ is shifted away. (d, g) The pump power applied to the link mode $m_{0}$ is increased to 173 $\mu \mathrm{W}$, which further shifts the $m_{0}$ mode, and it now completes the necklace at $\lambda^{+}$. In panels $\mathrm{e}-\mathrm{g}$, the dimmed modes and dotted lines represent low transmission, whereas bright colored modes and solid lines show increased transmission. See Supplementary Figures S1, S2, and S3 for additional data that provide proof of robustness of our reversible control. The data are provided as an open supplementary content. 

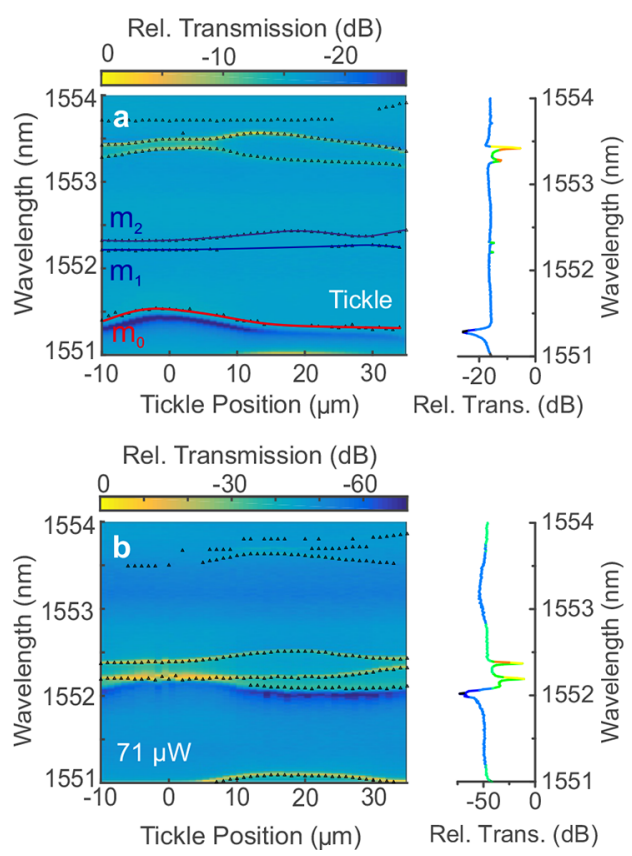
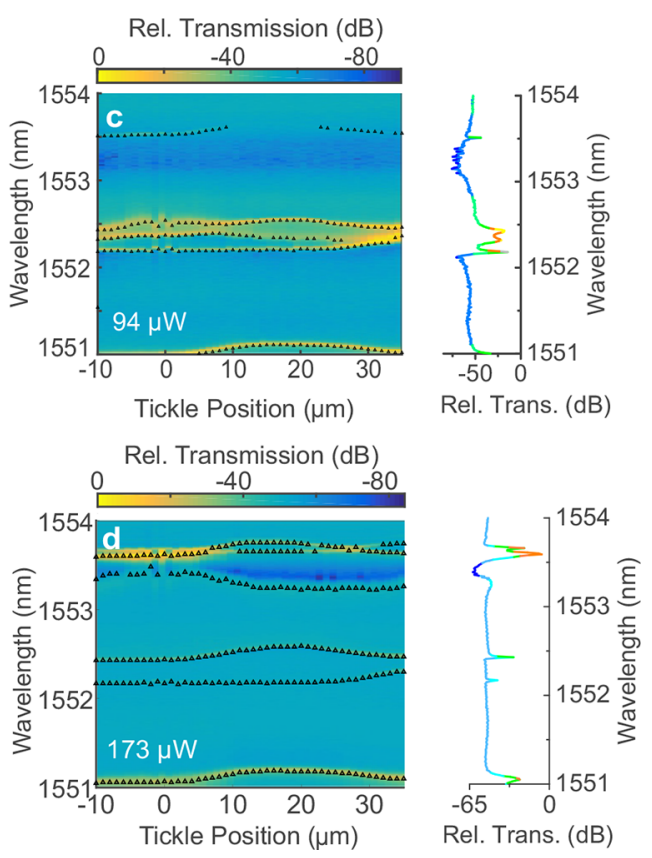

Figure 3. Control of the necklace states in a photonic crystal waveguide, mapped in wavelength and in position. The density plots show transmission versus wavelength and position of the tickle beam. The graphs at the side of each panel show relative transmission versus wavelength, taken at a tickle spot position at $35 \mu \mathrm{m}$. (a) The $m_{0}$ mode is located spatially using a tickle beam. The modes $m_{1}$ and $m_{2}$ are located at 1552.2 and $1552.4 \mathrm{~nm}$, respectively. The mode at $m_{1}$ is spatially centered at $29 \mu \mathrm{m}$, and $m_{2}$ is located at $18 \mu \mathrm{m}$. The pump beam is positioned at 0 , on top of $m_{0}$. In panels b-d the power is increased stepwise. (b) At $71 \mu \mathrm{W}, m_{0}$ weakly couples to $m_{1}$. (c) Strong coupling of $m_{0}$ to $m_{2}$. (d) The $m_{0}$ mode is decoupled from the necklace at $173 \mu \mathrm{W}$ pump power. The black and red solid bold curves are guides to the eye. See Supplementary Figure S5 for intermediate power steps.

(barrier) waveguide at $W_{0}=0.98 \sqrt{3} a$ is optimized for dispersion, whereas the $W_{1}=1.1 \sqrt{3} a$ is chosen so that its band edge is shifted considerably and transmits well in the region where the main waveguide possesses localized resonances. The measured quality factors of the localized modes in the photonic crystal waveguide range up to $10^{5}$.

Figure 2(a) shows transmission through our GaInP photonic crystal waveguide versus wavelength at various pump powers. One can observe two small transmission features, corresponding to modes labeled $m_{0}$ and $m_{0}^{*}$, that shift strongly with pump power. The other transmission features do not shift appreciably, which indicates that we have independent control over the resonance frequencies of localized modes (see Figure 2) thanks to the narrow spatial profile of our pump. A group of modes between 1552 and $1552.5 \mathrm{~nm}$ is not shifted in frequency, indicating negligible spatial overlap with the pump. However, the transmission of these modes is strongly increased when they become resonant with $m_{0}$. Similarly, the transmission of the modes between 1553.25 and $1553.75 \mathrm{~nm}$ is decreased, as they lose spectral overlap with mode $m_{0}^{*}$ and recover as they regain spectral overlap with mode $m_{0}$. The pump beam is kept on modes $m_{0}$ and $m_{0}^{*}$ during all the measurements. Before and after the tuning experiments we obtain reference spectra, shown as the very first and the last curves, to validate that there is no permanent change on the sample. Indeed the second reference spectrum is almost identical to the first reference spectrum. A small decrease in intensity is attributed to drift of coupling losses that only affect the total transmission amplitude and not the spectral position.

In Figure $2(b-d)$ we present salient features of the transmission spectra at a higher resolution and in $\mathrm{dB}$ scale, which shows the modulation depth more clearly. Figure 2(b) depicts a reference transmission spectrum. In the reference spectrum we detect weak transmission peaks in the wavelength region marked by $\lambda^{*}(1552$ to $1552.5 \mathrm{~nm})$ and stronger transmission in the region $\lambda^{+}(1553.25$ to $1553.75 \mathrm{~nm})$. Next, we shine $71 \mu \mathrm{W}$ of pump laser light on the mode $m_{0}$, which makes it resonant with the modes located at the $\lambda^{*}$ region. As a result, transmission at $\lambda^{*}$ is increased by $15 \mathrm{~dB}$ and transmission at $\lambda^{+}$is decreased to the noise level; see Figure 2 (c). Finally, the power on the $m_{0}$ mode is increased to 173 $\mu \mathrm{W}$, which brings $m_{0}$ into resonance with $\lambda^{+}$, and consequently the transmission at $\lambda^{+}$increases by $23 \mathrm{~dB}$, while the transmission at $\lambda^{*}$ decreases; see Figure $2(\mathrm{~d})$. While we are tuning the $m_{0}$ mode away from the $\lambda^{*}$ region, the spectral overlap of mode $m_{0}$, given the broad Fano profile, is still maintained up to a certain degree. Moreover, our method enables us to mark only the localized modes that we can identify in transmission. The system has more localized modes that are out of resonance, and we may not be able to resolve a transmission peak for these modes. As we tune a localized mode, more modes can possibly couple to the necklace and contribute to the transmission. For these reasons, the signal at the $\lambda^{*}$ region does not drop to noise level as in the $\lambda^{+}$region. The strong modulation of the transmission shows that we can control necklace states by independently controlling one of the necklace links.

A schematic model of these necklace states is shown in Figure 2(e-g). In Figure 2(e) we depict our interpretation of the reference spectrum: In this case the link mode $m_{0}$ is out of resonance, leading to low transmission. A spatially nearby $m_{0}^{*}$ mode enables a weak transmission at $\lambda^{+}$. When we tune the refractive index locally, the $m_{0}$ mode is tuned into resonance and completes the chain at $\lambda^{*}$, leading to an increased transmission. At the same time, the mode $m_{0}^{*}$ is pushed out of 
resonance with the necklace state at $\lambda^{+}$, decreasing transmission in that part of the spectrum. In the third step (Figure $2 \mathrm{~d}, \mathrm{~g}$ ) the power on the $m_{0}$ mode is increased to $173 \mu \mathrm{W}$, which induces a greater shift in wavelength. At this power level the $m_{0}$ mode decouples from the necklace state at $\lambda^{*}$ and completes the chain at $\lambda^{+}$. As a result, the transmission at $\lambda^{*}$ is decreased, while the chain at $\lambda^{+}$shows a high transmission. The independent control of one of the link modes in a necklace state enables us to switch the wavelength at which the sample becomes transmitting.

Detailed spatial information on the coupled modes is represented in Figure 3, showing a color map of the transmission versus frequency and tickle beam position. In Figure 3(a) only the tickle beam is present. The tickle beam induces a small index perturbation and that effectively shifts the mode by about $0.24 \mathrm{~nm}$. From the maxima of the tickleinduced wavelength shifts we locate mode $m_{0}$ at $0 \mu \mathrm{m}$, mode $m_{1}$ at $29 \mu \mathrm{m}$, and mode $m_{2}$ at $18 \mu \mathrm{m}$. From the fact that these modes are discrete (no overlap in frequency unless we tune) and that their mode profile is measured to be smaller than the system size spatially, we conclude they are localized through coherent random scattering, i.e, Anderson localized. From the spectrum in Figure 3(a) we see that the line shape of mode $m_{0}$ resembles a Fano profile, which arises from the interference of the discrete (localized) resonance with a transmission continuum; ${ }^{17-20}$ see Supplementary Figure S4. Next, we position the pump beam at position 0 , to spatially overlap with $m_{0}$, and tune the refractive index locally. The hybridization and the anticrossings of the localized modes ${ }^{21}$ are observed at the position of the linking mode. In Figure 3(b) we see that mode $m_{0}$ shifts in wavelength by $0.9 \mathrm{~nm}$, due to the pump and the tickle beam, and overlaps in wavelength with $m_{1}$. We increase the pump power to $94 \mu \mathrm{W}$, as shown in Figure 3 (c). Now, mode $m_{0}$ couples to mode $m_{2}$ strongly, as is apparent from the wide avoided crossing. Scanning the tickle beam on top of a pump provides us the means to measure the anticrossing width. Although the tickle beam induces a wavelength shift of $0.24 \mathrm{~nm}$ typically, we observe that when mode $m_{0}$ is at the vicinity of mode $m_{2}$, the shift induced by the tickle beam is much smaller $(0.05 \mathrm{~nm})$, as expected for coupled modes in an anticrossing. In Figure 3(c) the coupled modes obtain a flat spatial profile, which indicates the increased spatial size of the hybridized modes. When two modes weakly couple, the spatial mode profile gets broader in space due to hybridization. When the modes are strongly coupled, the modes repel each other in frequency, which results in shifting of the spectral position as well as an increase or decrease in transmission, as can be seen in Figure 3. In Supplementary Figure S5, we provide measurements at intermediate pump power levels. Finally, the pump power is increased to $173 \mu \mathrm{W}$ on the mode $m_{0}$. At this power level the $m_{0}$ mode is decoupled again; it is now at higher wavelength than modes $m_{1}$ and $m_{2}$.

The pump-dependent hybridization of modes demonstrates that we can locally tune a localized mode and couple it to other localized modes weakly or strongly. The coupling strength is determined by the spatial position of the modes. For instance, when we tune mode $m_{0}$ to the same frequency as mode $m_{1}$, we observe no avoided crossings since the distance between these modes is as large as $29 \mu \mathrm{m}$, making them weakly coupled. However, between modes $m_{0}$ and $m_{2}$, which are close to each other in space, a wide avoided crossing of $0.1 \mathrm{~nm}$ is measured.

Using spatial control of the intrinsically localized modes in a waveguide we form, or break, highly transmissive necklace states so that the transmission can be modulated at a given wavelength. The pump-tickle-probe method that we introduce here provide us the means to identify the coupling type, trace the anticrossing regime, and detect spatial mode profile changes of a collection of disordered high- $Q$ localized modes and extended necklace states. Multiple-resonant systems $s^{20,22}$ that we are able to control here are pronounced platforms to study fundamental physics ${ }^{23}$ and explore alloptical devices that involve slow light. ${ }^{24}$ In more complex geometries, controlling multiple localized modes in a necklace state via holographic patterns can enable routing light on chip in 2D optical networks by coherently coupling Anderson localized modes. ${ }^{7,25}$ This control of coupled narrow-band resonant modes is an essential step in the coupling of quantum light sources that can be embedded inside photonic crystal waveguides and which offer novel opportunities for creating multinode quantum networks. ${ }^{26-31}$

\section{ASSOCIATED CONTENT}

S Supporting Information

The Supporting Information is available free of charge on the ACS Publications website at DOI: 10.1021/acsphotonics.8b01038.

Supporting content (PDF)

Measurement data of Figure 2(a) in tabular form (XLSX)

\section{AUTHOR INFORMATION}

\section{Corresponding Author}

*E-mail: eyuce@metu.edu.tr.

ORCID $\odot$

Emre Yüce: 0000-0001-7808-1988

\section{Notes}

The authors declare no competing financial interest.

\section{ACKNOWLEDGMENTS}

We thank Willem L. Vos, Ad Lagendijk, Pepijn W. H. Pinkse, Henri Thyrrestrup, and Sanli Faez for useful discussions. This research was supported by ERC-pharos -279248 and Netherlands Organization for Scientific Research (Vici 68047618).

\section{REFERENCES}

(1) Koenderink, A. F.; Lagendijk, A.; Vos, W. L. Optical extinction due to intrinsic structural variations of photonic crystals. Phys. Rev. B: Condens. Matter Mater. Phys. 2005, 72, $153102-1-4$.

(2) Hughes, S.; Ramunno, L.; Young, J. F.; Sipe, J. E. Extrinsic Optical Scattering Loss in Photonic Crystal Waveguides: Role of Fabrication Disorder and Photon Group Velocity. Phys. Rev. Lett. 2005, 94, 033903.

(3) Sokolov, S.; Lian, J.; Yüce, E.; Combrié, S.; Rossi, A. D.; Mosk, A. P. Tuning out disorder-induced localization in nanophotonic cavity arrays. Opt. Express 2017, 25, 4598-4606.

(4) Anderson, P. W. Absence of Diffusion in Certain Random Lattices. Phys. Rev. 1958, 109, 1492-1505.

(5) Topolancik, J.; Ilic, B.; Vollmer, F. Experimental Observation of Strong Photon Localization in Disordered Photonic Crystal Waveguides. Phys. Rev. Lett. 2007, 99, $253901-1-4$.

(6) Mazoyer, S.; Hugonin, J. P.; Lalanne, P. Disorder-Induced Multiple Scattering in Photonic-Crystal Waveguides. Phys. Rev. Lett. 2009, 103, 063903.

(7) Sapienza, L.; Thyrrestrup, H.; Stobbe, S.; Garcia, P. D.; Smolka, S.; Lodahl, P. Cavity Quantum Electrodynamics with AndersonLocalized Modes. Science 2010, 327, 1352-1355. 
(8) Crane, T.; Trojak, O. J.; Vasco, J. P.; Hughes, S.; Sapienza, L. Anderson Localization of Visible Light on a Nanophotonic Chip. ACS Photonics 2017, 4, 2274-2280.

(9) Vasco, J. P.; Hughes, S. Statistics of Anderson-localized modes in disordered photonic crystal slab waveguides. Phys. Rev. B: Condens. Matter Mater. Phys. 2017, 95, 224202.

(10) Choi, S. H.; Byun, K. M.; Kim, Y. L. Lasing Interactions Disclose Hidden Modes of Necklace States in the Anderson Localized Regime. ACS Photonics 2018, 5, 881-889.

(11) Patterson, M.; Hughes, S.; Combrié, S.; Tran, N.-V.-Q.; De Rossi, A.; Gabet, R.; Jaouën, Y. Disorder-Induced Coherent Scattering in Slow-Light Photonic Crystal Waveguides. Phys. Rev. Lett. 2009, $102,253903$.

(12) Faggiani, R.; Baron, A.; Zang, X.; Lalouat, L.; Schulz, S. A.; Regan, B. O.; Vynck, K.; Cluzel, B.; de Fornel, F.; Krauss, T. F.; Lalanne, P. Lower bound for the spatial extent of localized modes in photonic-crystal waveguides with small random imperfections. Sci. Rep. 2016, 6, 27037.

(13) Riboli, F.; Caselli, N.; Vignolini, S.; Intonti, F.; Vynck, K.; Barthelemy, P.; Gerardino, A.; Balet, L.; Li, L. H.; Fiore, A.; Gurioli, M.; Wiersma, D. S. Engineering of light confinement in strongly scattering disordered media. Nat. Mater. 2014, 13, 720-725.

(14) Pendry, J. B. Quasi-extended electron states in strongly disordered systems. J. Phys. C: Solid State Phys. 1987, 20, 733.

(15) Sokolov, S.; Lian, J.; Yüce, E.; Combrié, S.; Lehoucq, G.; Rossi, A. D.; Mosk, A. P. Local thermal resonance control of GaInP photonic crystal membrane cavities using ambient gas cooling. Appl. Phys. Lett. 2015, 106, 171113.

(16) Lian, J.; Sokolov, S.; Yüce, E.; Combrié, S.; Rossi, A. D.; Mosk, A. P. Measurement of the profiles of disorder-induced localized resonances in photonic crystal waveguides by local tuning. Opt. Express 2016, 24, 21939-21947.

(17) Fano, U. Effects of Configuration Interaction on Intensities and Phase Shifts. Phys. Rev. 1961, 124, 1866-1878.

(18) Galli, M.; Portalupi, S. L.; Belotti, M.; Andreani, L. C.; Faolain, L. O.; Krauss, T. F. Light scattering and Fano resonances in high-Q photonic crystal nanocavities. Appl. Phys. Lett. 2009, 94, 071101.

(19) Miroshnichenko, A. E.; Flach, S.; Kivshar, Y. S. Fano resonances in nanoscale structures. Rev. Mod. Phys. 2010, 82, $2257-2298$.

(20) Lian, J.; Sokolov, S.; Yüce, E.; Combrié, S.; Rossi, A. D.; Mosk, A. P. Fano lines in the reflection spectrum of directly coupled systems of waveguides and cavities: measurements, modeling and manipulation of the Fano asymmetry. Phys. Rev. A: At., Mol., Opt. Phys. 2017, 96, 033812.

(21) Bliokh, K. Y.; Bliokh, Y. P.; Freilikher, V.; Genack, A. Z.; Sebbah, P. Coupling and Level Repulsion in the Localized Regime: From Isolated to Quasiextended Modes. Phys. Rev. Lett. 2008, 101, 133901.

(22) Yariv, A.; Xu, Y.; Lee, R. K.; Scherer, A. Coupled-resonator optical waveguide: a proposal and analysis. Opt. Lett. 1999, 24, 711713.

(23) Hafezi, M.; Mittal, S.; Fan, J.; Migdall, A.; Taylor, J. M. Imaging topological edge states in silicon photonics. Nat. Photonics 2013, 7, 1001.

(24) Krauss, T. F. Why do we need slow light? Nat. Photonics 2008, 2, 448.

(25) Ren-Jye, S.; Efetov Dmitri, K.; Gabriele, G.; Cheng, P.; Chung, F. K.; Dirk, E. Nanophotonics 2017, DOI: 10.1515/nanoph-20160172.

(26) Greentree, A. D.; Tahan, C.; Cole, J. H.; Hollenberg, L. C. L. Quantum phase transitions of light. Nat. Phys. 2006, 2, 856-861.

(27) Fushman, I.; Waks, E.; Englund, D.; Stoltz, N.; Petroff, P.; Vučković, J. Ultrafast nonlinear optical tuning of photonic crystal cavities. Appl. Phys. Lett. 2007, 90, 091118.

(28) Kimble, H. J. The quantum internet. Nature (London, U. K.) 2008, 453, 1023-1030.

(29) Englund, D.; Majumdar, A.; Bajcsy, M.; Faraon, A.; Petroff, P.; Vučković, J. Ultrafast Photon-Photon Interaction in a Strongly
Coupled Quantum Dot-Cavity System. Phys. Rev. Lett. 2012, 108, 093604.

(30) Stockill, R.; Stanley, M. J.; Huthmacher, L.; Clarke, E.; Hugues, M.; Miller, A. J.; Matthiesen, C.; Le Gall, C.; Atatüre, M. Phase-Tuned Entangled State Generation between Distant Spin Qubits. Phys. Rev. Lett. 2017, 119, 010503.

(31) Lodahl, P. Quantum-dot based photonic quantum networks. Quantum Science and Technology 2018, 3, 013001. 